Nouveaux cahiers de la recherche en éducation

Risiko oder Gefahr, Bedarfe oder Bedürfnisse? Zur diskursiven Gleichzeitigkeit von sich widersprechenden Grundideen bei der Entwicklung bildungspolitischer Projekte im Kontext Internationaler Organisationen

Élèves à risque ou en péril, en détresse ou à besoins spécifiques? Sur la simultanéité discursive d'idées contradictoires développées lors de l'élaboration de projets éducatifs par des organisations internationales

At Risk or in Danger, in Need or with Requirements? On the discursive simultaneity of contradictory ideas in the course of the development of educational projects in context of International Organisations

¿Alumnos en riesgo o en peligro, desamparados o con necesidades específicas? Sobre la simultaneidad discursiva de ideas contradictorias desarrolladas durante la elaboración de proyectos educativos por organismos internacionales

Florian Kiuppis

Volume 17, numéro 1, 2014

Traductions

Translations

URI : https://id.erudit.org/iderudit/1027323ar

DOI : https://doi.org/10.7202/1027323ar

Aller au sommaire du numéro

Éditeur(s)

Faculté d'éducation, Université de Sherbrooke

ISSN

1911-8805 (numérique)

Découvrir la revue

Citer cet article

Kiuppis, F. (2014). Risiko oder Gefahr, Bedarfe oder Bedürfnisse? Zur diskursiven Gleichzeitigkeit von sich widersprechenden Grundideen bei der Entwicklung bildungspolitischer Projekte im Kontext Internationaler Organisationen. Nouveaux cahiers de la recherche en éducation, 17(1), 93-120. https://doi.org/10.7202/1027323ar
Résumé de l'article

Le texte traite d'un cas de transformation des connaissances reflétant un processus de " traduction d'idées ", dans le cadre des négociations au cours du déroulement d'un projet entre le Centre pour la recherche et l'innovation dans l'enseignement (CERI) de l'OCDE et d'autres acteurs impliqués dans son élaboration auprès d'enfants et de jeunes défavorisés. L'accent est mis sur le projet Children and Youth at Risk, réalisé dans les années 1990 et dont l'objectif était de garantir l'accès à l'éducation pour les apprenants considérés comme étant les plus défavorisés: principalement les enfants et les jeunes vivant dans la pauvreté et/ou appartenant à des minorités ethniques. Les données ont été recueillies à partir de documents d'archives réunis au siège de l'OCDE à Paris. La méthode appliquée est l'analyse de contenu. 


\section{Risiko oder Gefahr, Bedarfe oder Bedürfnisse? Zur diskursiven Gleichzeitigkeit von sich widersprechenden Grundideen bei der Entwicklung bildungspolitischer Projekte im Kontext Internationaler Organisationen}

\section{FLORIAN KIUPPIS}

Lillehammer University College

\section{Zusammenfassung}

Der Text befasst sich mit einem Fall der Transformation von Wissen, an dem die Übersetzung von Ideen in jenem Kontext deutlich wird, in dem sich die Aushandlung der Grundzüge eines Projekts für benachteiligte Kinder und Jugendliche zwischen dem Center for Educational Research and Innovation der OECD und anderen an dieser Entwicklung beteiligten Akteuren abspielt. Im Fokus steht das Projekt Children and Youth at Risk, das im Verlauf der 1990er-Jahre mit dem Ziel durchgeführt wurde, insbesondere jenen Gruppen Zugang zu Bildungsangeboten zu gewähren, die als am meisten benachteiligt galten: vor allem Kinder, die in Armut leben und ethnische Minderheiten. Als Quellenbasis für die Untersuchung dienen Dokumente aus den Archivbeständen der OECD in Paris, die inhaltsanalytisch ausgewertet wurden.

Schlagworte: Vergleichende Bildungswissenschaft, Internationale Organisationen, OECD, Soziologie der Übersetzung; Bedeutungen. 
Elèves à risque ou en péril, en détresse ou à besoins spécifiques?

Sur la simultanéité discursive d'idées contradictoires développées lors de l'élaboration de projets éducatifs par des organisations internationales

\section{Résumé}

Le texte traite d'un cas de transformation des connaissances reflétant un processus de "traduction d'idées", dans le cadre des négociations au cours du déroulement d'un projet entre le Centre pour la recherche et l'innovation dans l'enseignement (CERI) de I'OCDE et d'autres acteurs impliqués dans son élaboration auprès d'enfants et de jeunes défavorisés. L'accent est mis sur le projet Children and Youth at Risk, réalisé dans les années 1990 et dont l'objectif était de garantir l'accès à l'éducation pour les apprenants considérés comme étant les plus défavorisés: principalement les enfants et les jeunes vivant dans la pauvreté et/ou appartenant à des minorités ethniques. Les données ont été recueillies à partir de documents d'archives réunis au siège de l'OCDE à Paris. La méthode appliquée est l'analyse de contenu.

Mots-clés: éducation comparée, organisations internationales, OCDE, sociologie de la traduction, sens.

\section{At Risk or in Danger, in Need or with Requirements? On the discursive simultaneity of contradictory ideas in the course of the development of educational projects in context of International Organisations}

\section{Abstract}

The text deals with a case of the transformation of knowledge which reflects "translations of ideas" in the context of negotiations during the development of a project for disadvantaged children and youths, run by the Center for Educational Research and Innovation (OECD). The focus is on the project Children and Youth at Risk, carried out throughout the 1990's, and connected with the goal to guarantee access to education for those learners considered most disadvantaged: primarily children and youth living in 
poverty, and/or belonging to ethnic minorities. Data is drawn from archival documents gathered in the OECD Headquarters in Paris. Method applied is content analysis.

Key words: comparative Education, International Organisations, OECD, Sociology of Translation, meanings.

\section{¿Alumnos en riesgo o en peligro, desamparados o con necesidades específicas? Sobre la simultaneidad discursiva de ideas contradictorias desarrolladas durante la elaboración de proyectos educativos por organismos internacionales}

\section{Resumen}

Se trata de un caso de transformación de los conocimientos que refleja un proceso de "traducción de ideas", en el marco de negociaciones durante el desarrollo de un proyecto entre el Centro para la Investigación e Innovación Educativa (CIIE) de la Organización para la Cooperación y el Desarrollo Económicos (OCDE) y con otros actores involucrados en su elaboración con niños y jóvenes desfavorecidos. El acento está puesto en el proyecto Children and Youth at Risk realizado en los años 1990 y cuyo objetivo era garantizar el acceso a la educación a los alumnos considerados como siendo los más desfavorecidos: principalmente los niños y los jóvenes que viven en la pobreza y/o que pertenecen a minoridades étnicas. Los datos fueron colectados a partir de documentos de archivos reunidos en la sede de la OCDE en París. Se llevó a cabo el estudio con el método de análisis de contenido.

Palabras clave: educación comparada, organizaciones internacionales, OCDE, sociología de la traducción, significado. 


\section{Einleitung}

Der OECD wird mitunter die Eigenschaft zugeschrieben, viel Wert darauf zu legen, Konsens mit ihren Peers und Competitors (Scott, 1994, S. 43) zu erzielen. So bringen zum Beispiel Nik Theodore und Jamie Peck - deren Arbeiten zu Globalisierung in letzter Zeit häufig im Kontext der Vergleichenden Bildungswissenschaft herangezogen werden (Dale, 2012; Robertson, 2012; Verger et al., 2013) - treffend auf den Punkt, die OECD sei eine consensus-finding organization [...], not so much a unilateral maker but a multilateral mediator of policy (Theodore \& Peck, 2012, S. 20). In diesem Sinne wird die OECD in den Bildungswissenschaften mitunter mit "soft governance" assoziiert (Mausethagen, 2013) und als eine Organisation beschrieben, der es nicht so sehr um radikale Innovationen sondern vielmehr um die Definition eines gemeinsamen Nenners in einem koordinativen Diskurs gehe. In diesem Zusammenhang wird verständlich, was Anja P. Jakobi (2007) für den deutschsprachigen Raum am Beispiel "Lebenslangen Lernen" exemplifiziert hat: die OECD verfüge kaum über direkte Steuerungsmechanismen, etwa über die Möglichkeit der Regulierung, und arbeite vor allem mit "diskursiven Instrumenten", zum Beispiel mit Überzeugung (Jakobi, 2007, S. 166; vgl. hierzu auch Rinne \& Ozga, 2011, sowie Centeno, 2011).

Im vorliegenden Beitrag zum Themenheft "Übersetzungen" wird dieses Wort nicht linguistisch, sondern wissens- bzw. organisationssoziologisch verstanden. Anhand von Dokumenten aus den einschlägigen Bibliotheks- und Archivbeständen der OECD in Paris erfolgt eine theoretisch fundierte Auseinandersetzung mit der Frage, was mit solchen Ideen geschieht, die im Rahmen der Entwicklung bildungsorganisatorischer Projekte von Seiten der OECD in bildungspolitische Diskurse eingebracht werden, aber in den Mitgliedsländern nicht auf positive Resonanz stossen. Aus der Organisationsforschung bieten sich für die Behandlung dieses Themas Arbeiten an, die sich mit der Frage befassen "how diffusion can fail» (Jonsson, 2009), wie mit anderen Worten mit Blick auf die Ebene der intendierten Adressaten pädagogischer Ideen (im hier vorliegenden Fall 24 - der heute 34 Mitgliedstaaten der OECD) zu erklären ist, warum sich manche Wissensbestände «erfolgreich" verbreiten können, d.h. bei bestimmten Akteuren auf nationaler Ebene auf entsprechend 
positive Resonanz stossen, und wie wiederum andere abgelehnt oder ignoriert werden. In Anlehnung an die aufgeführte Literatur lassen sich hier vorab verschiedene Szenarien hypothetisch annehmen: zum Beispiel, dass die OECD durch Nutzung des Instruments der Überzeugung versucht, ihre Peers und Competitors auf ihre eigene Lesart zu spezifischen Themen einzustimmen und kollektive Zielvorstellungen anzustreben, die eigenen Motiven entsprechen; oder dass sich die OECD in ihrer Rhetorik allmählich den von Seiten ihrer Mitgliedstaaten an sie gestellten Anforderungen bzw. Vorstellungen von Peers anpasst, evtl. mit der Konsequenz, dass Projektbeschreibungen zunehmend die Handschrift von am Kommunikationsprozess beteiligten Ministerien und anderen Gruppen annimmt.

Hier geht es um einen Fall, an dem sich auf der Ebene des Absenders theoretisierten Wissens (OECD) abzeichnet, dass bestimmte Ideen, die in Projektbeschreibungen enthalten waren, in übersetzter und insofern transformierter Form zurückkehren, offenbar weil sie sich mit kontextspezifischen Erfordernissen auf nationaler Ebene als nicht kompatibel oder als nicht wünschenswert erwiesen. In Folge solcher hier organisations- und wissenssoziologisch konzeptualisierter "Übersetzungen» kommt es im Rahmen der Entwicklung des in diesem Beitrag exemplarisch hervorgehobenen bildungspolitischen Projekts zu diskursiven Gleichzeitigkeiten sich widersprechender Grundideen bei der Entwicklung bildungspolitischer Projekte, in diesem Fall im Zusammenhang mit dem Sachverhalt bzw. Sozialen Problem Children and Youth at Risk.

Seinem thematischen Zuschnitt nach zu urteilen, lässt sich dieser Beitrag einem Corpus von Arbeiten in der Vergleichenden Bildungswissenschaft zuordnen, das als historische Globalisierungsforschung zusammenzufassen ist. Er ist vor allem durch solche Studien inspiriert, die sich im Allgemeinen mit der Internationalisierung pädagogischen Wissens und im Besonderen mit Prozessverläufen der Transformation desselben befassen - sei es auf der globalen Ebene als "reisendes" oder auf der lokalen Ebene als "gereistes" Wissen. Die Quellen aus den Archiven der OECD, wobei es sich um alle für das Thema "Educational Disadvantage» relevanten Dossiers des komplett archivierten Dokumentenbestands des Center for Educational Research and Innovation (CERI) der OECD für den Zeitraum bis zum Jahr 2001 handelt, wurden inhaltsanalytisch ausgewertet (vgl. Krippendorff, 2012). 
Dieser Prozess vollzog sich insbesondere in Heranziehung der Leitfrage, inwiefern sich die Stellungnahmen der OECD im Kontext des Projekts Children and Youth at Risk von jenen anderer Akteure unterscheiden. Somit handelt es sich bei diesem Beitrag weniger um eine rekonstruktive Prozessanalyse, die nachzuvollziehen versuchen würde, wie eine Internationale Regierungsorganisation Implementierungsprozesse begleitet und nach ihrem Erfolg trachtet (für Beispiele hierzu vgl. z.B. Kiuppis, 2007; 2014a). Die Behandlung des Themas findet hier also nicht im Sinne eines um minutiöse Aufschlüsselung von Abstimmungsund Entscheidungsprozessen bemühten Nachvollzugs der komplexen Kommunikationsund Interaktionsgefüge zwischen verschiedenen Akteurstypen und Akteursgruppen statt. Vielmehr erfolgt hier eine Thematisierung der Pluralität von im unmittelbaren Kontext des CERI gleichzeitig koexistierenden Verständnissen und Bedeutungen von at risk und success sowie damit assoziierter Sozialer Probleme, Sachverhalte und imaginierter Lösungen. Was durch die hier gewählte Vorgehensweise der Analyse nicht in den Blickwinkel gerät, sind jene Abläufe, die von der Kulturwissenschaftlerin Susan Wright (1998, S. 9) als "contested processes of meaning making» bezeichnet werden. Diese - wie auch Konstruktionen, die von auf Ebene der OECD-Mitgliedstaaten vorgelebten innovativen Beispielen abstrahieren und insofern de-kontextualisiert sind - können hier nur mutmasslich angenommen werden.

\section{Kontextualisierung in den Bildungswissenschaften}

Die Perspektive, in der pädagogisches Wissen analysiert wird, das "auf Reisen geht" (Schulte, 2008), ist in den Bildungswissenschaften zuallererst im Rahmen der sogenannten Transferforschung in "Diffusionsanalysen" zu finden, die vor allem in der Vergleichenden Bildungswissenschaft im anglo-amerikanischen Raum verbreitet sind. Darin wird typischerweise davon ausgegangen, dass pädagogische Modelle und Konzepte sich von der globalen Ebene - mehr oder weniger linear oder "zirkulär" - zu lokalen Kontexten bewegen bzw. transportiert werden (Carnoy \& Rhoten, 2002; Dale, 2005; Nóvoa \& YarivMashal, 2003).

Zweitens geben Arbeiten aus Forschungszusammenhängen zum Themenkomplex Policy Borrowing and Lending wichtige - zumal theoriebasierte und insofern konzeptionell 
aufschlussreiche - Einblicke in Kontexte, in denen pädagogische Modelle implementiert werden, die anderswo entwickelt wurden (vgl. u.a. Phillips \& Ochs, 2004; Steiner-Khamsi, 2006; Steiner-Khamsi \& Waldow, 2012). Die Perspektive, in der "gereistes Wissen" analysiert wird, ist in der Vergleichenden Bildungswissenschaft in der Regel in "Rezeptionsanalysen" zu finden. Forschungen aus dieser Richtung beziehen sich üblicherweise auf die Fragen, wie Akteure und ihre Interessen und Zielsetzungen in (sub-)nationalen Kontexten mit Ideen verknüpft sind, die auf globaler Ebene auf "Reisen» waren, und welche Umwandlungsprozesse diese Ideen durchlaufen, wenn sie als von Reisen bereichert wiederkehrendes Wissen auf der lokalen Ebene eintreffen.

In diesem Zusammenhang stellen sich drittens besonders solche Arbeiten als für die hier eingenommene Analyseperspektive relevant heraus, in denen Fälle unterschiedlicher Interpretationen derselben Konzepte in verschiedenen Kontexten untersucht werden (vgl. u.a. Takayama, 2012; Ozga, 2005). Dort geht es um Fälle, in denen "gereistes" Wissen im Zusammenhang mit Kulturen und Praktiken auf (sub)nationaler Ebene verändert wird und es somit einerseits zu bestimmten lokalen Versionen dieses Wissens kommt und andererseits zu dem Phänomen, dass alten Strukturen neue Bedeutungen eingehaucht werden.

Als von hervorgehobener Wichtigkeit für das hier behandelte Thema haben sich viertens jene Studien erwiesen, die Anschauung bieten und empirisch Aufschluss darüber geben, wie es im Zuge der weltumgreifenden Verbreitung und jeweils kontextspezifischen Umsetzung von pädagogischen Modellen und Konzepten, unter Einwirkung unterschiedlicher Akteure zu ihrer Veränderung kommt. Als besonders aufschlussreich stellen sich in diesem Zusammenhang Studien heraus, in denen zum einen die Annahme der klassischen Diffusionsforschung hinterfragt wird, wonach zu untersuchende Wissensbestände, die von der globalen Ebene in Richtung lokaler Kontexte "reisen", dabei so lange unverändert bleiben, bis sie an ihrem Ziel (d.h. dem zur Analyse stehenden Kontext) angekommen sind (vgl. Cowen, 2009; Waldow, 2009). Zum anderen sind in diesem Kontext Arbeiten zu nennen, in denen mit der Tradition der klassischen Diffusionsforschung gebrochen wird, den Fokus der Analyse in erster Linie oder sogar ausschliesslich auf die Diffusion von Strukturen zu richten ohne dabei die Motivation der an der Verbreitung (und Veränderung) jeweiligen 
Wissens beteiligten Akteure zu berücksichtigen (vgl. hierzu u.a. Roldán-Vera \& Schupp, 2006; Caruso, 2008).

Auf diese vier Corpora von Bezugsliteratur in den Bildungswissenschaften lässt sich die im Zusammenhang mit sich veränderndem Wissen im Zuge seiner Diffusion vom britischen Bildungsforscher Robert Cowen stammende Formel as it moves, it morphs (Cowen, 2009, S. 315) anwenden. Diese stellt sich auch für den vorliegenden Beitrag insofern als bedeutsam heraus, als das ihm zugrundeliegende Erkenntnisinteresse auf den Aspekt der Transformation von Wissen im Verlauf seiner Entwicklung und Verbreitung abzielt. In diesem Text sind es allerdings nicht Prozesse der Verwandlung sondern vielmehr durch Akteure aktiv und vermutlich bewusst vorgenommene Umwandlungen des Wissens, die als "Übersetzungen" interpretiert werden. Also geht es hier in erster Linie um solche Umwandlungen von Wissen, die an Ideen aktiv vollzogen werden (vielmehr als: sich an Ideen vollziehen). Entsprechend ist für den vorliegenden Text davon auszugehen, dass die OECD, die das Projekt Children and Youth at Risk federführend entwickelt und verbreitet hat, im Laufe der Konkretisierung der ihm zugrundeliegenden Idee gleichsam zum rezipierenden Akteur wird. So werden dieser Internationalen Organisation z.B. von Seiten der Mitgliedsländer Erwartungen und Erfahrungen jener Akteure auf staatlicher sowie lokaler Ebene zugetragen, die das Projekt je nach Spezifika in nationalen Kontexten unterschiedlich interpretieren und einordnen. Insofern wird der Thematisierung der "Übersetzung" von Ideen die Annahme zugrundegelegt, dass es sich hier um die Konstruktion eines von konkret praktizierten Vorbildern auf nationaler Ebene abstrahierenden - in diesem Sinne de-kontextualisierten - imaginierten Projekts handelt und das Wissen, das im Rahmen der OECD erarbeitet wird, in der Regel bereits vielfach gebrochene Bedeutungen mit sich bringt, wenn es in nationalstaatlichen Kontexten (wieder) ankommt, um dort weitere rekontextualisierende Umdeutungen zu durchlaufen (vgl. u.a. Schriewer 2007; Steiner-Khamsi \& Quist, 2000, S. 275).

Vor diesem Hintergrund wird deutlich, dass für die Charakterisierung der hier skizzierten Arbeit der aus der Organisationsforschung stammende Leitsatz to transfer is to transform (Gherardi \& Nicolini, 2000, S. 19) gleichwohl zutreffender ist als Bob Cowens "as it moves, it morphs» (2009, S. 315), betont jener vielmehr die aktive Einwirkung von Akteuren auf die 
Veränderung des zu analysierenden Wissens. Die in den Bildungswissenschaften bekannte Kernthese der Übersetzung, dass neues "globales" Wissen mit dem Vorwissen von "lokalen" Rezipienten verknüpft wird und somit ein Wissensbestand transformiert und beizeiten völlig anders weitergegeben wird als ursprünglich gemeint, wird in diesem Beitrag erweitert. So grenzt sich diese Arbeit kritisch zu jenem grössten Teil bildungswissenschaftlicher Analysen Internationaler Organisationen $a b$, die diesen Untersuchungsgegenstand als "Makro"-Ebene globaler Verflechtungen behandeln. Die hier repräsentierte Studie fokussiert hingegen die "Mikro"-Ebene der Binnenstrukturen Internationaler Organisationen, nimmt also dezidiert eine intern-differenzierende Perspektive auf dieselben ein. Internationale Organisationen werden - in Begriffen der neo-institutionalistischen world culture-Theorie gesprochen nicht als "Skript»-Geber, sondern aufgrund ihrer Bindungen an bestimmte Reglements, Erwartungen und Vorgaben selbst als "scripted" unterstellt. Mit dieser Sichtweise, die den Folgen der Einwirkungen einzelner Akteure bei der Entwicklung des Projekts Children and Youth at Risk grosse Bedeutung beimisst, wird im vorliegenden Beitrag zugleich fallbasiert und theoretisch der Frage nachgegangen, wie solche Ideen einer neo-liberalen Wirtschaft, die von der OECD in den bildungspolitischen Diskurs eingebracht werden, beständig in ihrem Bedeutungsumfang und hinsichtlich ihrer Grenzen ausgehandelt und immer wieder neu bestimmt werden. Für diese Prozesse wird angenommen, dass die verschiedenen Haltungen, Meinungen und Positionierungen zum Sinn und Zweck dieses Projekts an andere bildungspolitische Programmatiken und Konzepte auf der Ebene Internationaler Organisationen und ihrer Rhetoriken, aber auch an nationale und lokale Diskurse in unterschiedlich fortgeschrittenen Gesellschaften anschlussfähig gehalten werden.

Obgleich sich aus der bei der Dokumentenanalyse eingenommenen Perspektive nicht die "Reiseroute" jeweiliger Wissensbestände im Detail verfolgen lässt, kann anhand der Quellen nachvollzogen werden, welche veränderungen an dem reisenden und gereisten Wissen vollzogen und welche Umstrukturierungen sich im Zuge der Auseinandersetzung mit dem aus unterschiedlichen Richtungen einkehrenden (mitunter "wiederkehrenden", durch Erfahrungen "bereicherten") Wissen auf der Ebene des Feldes ergeben bzw. auf der Ebene der im Fokus stehenden Organisation vorgenommen werden. Insofern stellt der in der vorliegenden Arbeit analysierte Kontext eine Schnittstelle für zirkulierende pädagogische 
Ideen und Konzepte dar, an der die analytische Differenzierung, welche Aspekte der jeweiligen hier verhandelten Wissensbestände aus "globalen" oder "lokalen" Kontexten kommen, schlechterdings nicht möglich ist. Da im hier präsentierten Fall, auf Grundlage der thematischen Schwerpunktsetzung und Datenlage, das Wissen nicht auf seiner "Reise" zwischen der OECD und anderen Akteuren (z.B. ihren Mitgliedstaaten) verfolgt werden kann, konzentriert sich die Analyse auf die in den Archivmaterialien deutlich werdenden Unterschiede in der Rahmung der im fokussierten Kontext kollektiv angegangenen Sozialen Probleme.

\section{Theoretische Annahmen zur «Übersetzung” von Ideen}

Die analytische Hervorhebung der Differenzen in der Rahmung des Themas Children and Youth at Risk durch verschiedene Akteure im Allgemeinen und in den Bedeutungen bestimmter Wörter im Besonderen erfolgt in Heranziehung eines Verständnisses von “Übersetzung», wie es - in Anlehnung an den französischen Philosophen Michel Serres (Serres, 1974) - von Michel Callon in die Soziologie übernommen (Callon, 1975), von Bruno Latour und anderen Vertretern der sogenannten Actor-Network-Theory (zu denen auch Callon gehört) in den Sozialwissenschaften weiterentwickelt (Latour, 2005; Callon, 1986; Law, 1992) und in erster Linie von Barbara Czarniawska in die Organisationsforschung vor allem in den sogenannten Skandinavischen Institutionalismus - übertragen wurde (Czarniawska \& Sevón, 1996). “Übersetzung» wird im vorliegenden Text also nicht im linguistischen Sinne als Übertragung von einer Sprache in eine andere verstanden, sondern in Anlehnung an ein in der neo-institutionalistischen Organisationsforschung inzwischen etabliertes Theoriemittel, demzufolge im Zusammenhang mit der Analyse von Aushandlungsprozessen pädagogischer Modelle und Ideen theoretisch davon ausgegangen wird, dass [t]he perceived attributes of an idea, the perceived characteristics of a problem and the match between them are all created, negotiated or imposed during [a] collective translation process (Czarniawska \& Joerges, 1996, S. 25). Die Frage nach der so verstandenen "Übersetzung» von Ideen bringt somit das Erfordernis mit sich, die Ebene der Bedeutung von Wörtern mitzubedenken. In Heranziehung des theoretischen Konzepts der «Übersetzung» geht es hier mit anderen Worten um das Auftauchen unterschiedlicher Bedeutungen von Wörtern 
in einem bestimmten organisationalen Kontext, wobei Bedeutungen, unter Verweis auf die Spätphilosophie Ludwig Wittgensteins, als der "Gebrauch [eines jeweiligen Wortes] in der Sprache» verstanden wird (Wittgenstein, 2001 [1936-1946]). Diesem konzept-basierten Verständnis von «Übersetzung» zufolge, das sich von der Nutzung des Begriffs translation als Metapher in der Vergleichenden Bildungswissenschaft unterscheidet (vgl. z.B. Ball, 1998; Broadfoot, 2000), «übersetzen» Akteure Elemente der innen zur Umsetzung nahegelegten "Skripts» mitsamt den darin eingeschriebenen gesellschaftlichen Erwartungen in Handlungen, indem sie die abstrakten Logiken, die in diesen kulturellen Vorlagen codiert sind, interpretieren und das darin enthaltene Wissen transformieren (vgl. u.a. Hasse \& Krücken, 2009). In Hinblick auf bestimmte Soziale Probleme und Sachverhalte kommt es somit akteursübergreifend zu einer Vielzahl an Umsetzungsvarianten diffundierender pädagogischer Ideen, deren Form und Inhalt erhebliche Unterschiede aufweisen.

Der Norwegische Sozialwissenschaftler Kjell Arne Røvik hat die Annahmen von Bruno Latour und von Vertreterinnen und Vertretern des Skandinavischen Institutionalismus nochmal mehr für Mikroanalysen zugänglich gemacht, indem er «Übersetzung» nicht in erster Linie als Weitergabe und Transformation von Wissensbeständen zwischen Organisationen begreift, sondern auf Prozesse innerhalb von Organisationen anwendet. Dabei bezieht Røvik sich - im Sinne von "interpersonal diffusion» (Strang \& Soule, 1998, S. 268) - auf die Umwandlung von Wissen, die er als "more or less deliberate» (Røvik, 2011, S. 642) bezeichnet. Die Charakterisierung solcher Prozesse als nicht unbedingt absichtlich erfolgende, eröffnet für die Analyse die Möglichkeit, in Heranziehung von Røviks Arbeiten auch jene Prozesse als "Übersetzungen» von Ideen zu klassifizieren, bei denen die Datenlage keinen Aufschluss darüber gibt, inwieweit sie sich absichtlich, bewusst oder willentlich vollziehen. Den Beitrag von Organisationen im Rahmen der Entwicklung von Ideen - auf welchen von Latour nicht weiter eingegangen wird - fasst Røvik wie folgt zusammen: [Organisations] actively translate and transform [ideas], sometimes making them more suitable as problem-solving tools (ebd., S. 637). ${ }^{1}$

1 Dass Røvik Übersetzung als Transformation definiert, hier aber eine Unterscheidung zwischen «translate» und "transform" vornimmt, ist nicht ganz stimmig. Der Organisationstheoretiker John Damm Scheuer weist bei seiner kritischen Würdigung von Røviks Ansatz darauf hin, dass [w] hen Røvik is pointing out that an idea might be imitated, combined or remelted, he is also implying that an idea may be translated in a way that is more or less in accordance with an original idea (Scheuer, 2006, S. 11).. 
Den Fokus bei der Analyse der «Übersetzungen» von Wissen - wie Røvik, mit Blick auf Kommunikationsprozesse innerhalb von Organisationen - auf die Art und Weise des Umgangs einzelner Organisationen mit Ideen zu richten statt - wie Latour, von ausserhalb auf die Weitergabe von Ideen von Akteur zu Akteur, entspricht im Grossen und Ganzen der empirischen Schwerpunktsetzung im folgenden Artikel. Røviks Arbeiten zum sogenannten "organizational idea-handling" (Røvik, 2011, S. 631) bieten für die hier eingenommene Perspektive wichtige Hilfsmittel an, um die Archivmaterialien mit Blick auf die Frage erschliessen zu können, wie Organisationen mit bestimmten Ideen umgehen und diese bearbeiten. "Handling" bezieht sich hier auf [...] the various ways organizations relate to and act towards [...] ideas. (ebd., S. 631). Røvik wirft in diesem Zusammenhang einige wichtige Fragen auf, die sich auch in vielen sogenannten Rezeptionsanalysen innerhalb der Vergleichenden Bildungswissenschaft stellen, so beispielsweise: What happens to ideas after adoption - are they decoupled from or translated into practice, and to what extent do ideas "work" and have longlasting effects on organizations? (Røvik, 2011, S. 631).

Tabelle: Ausgewählte theoretische Referenzen zum Konzept der «Übersetzung»

\begin{tabular}{|ll}
\hline Michel Serres (1974) & $\begin{array}{l}\text { Entwicklung des Konzepts (franz.: traduction) } \\
\text { Michel Callon (1975) }\end{array}$ \\
$\begin{array}{l}\text { Anwendung des Konzepts in der Soziologie } \\
\text { (franz.: traduction) }\end{array}$ \\
$\begin{array}{l}\text { Bruno Latour (1986; 2005); Michel Callon (1986); John Law } \\
\text { (1992) }\end{array}$ & $\begin{array}{l}\text { Weiterentwicklung des Konzepts in der Actor- } \\
\text { Network-Theory (engl.: translation) }\end{array}$ \\
$\begin{array}{l}\text { Barbara Czarniawska \& Bernward Joerges (1996); Barbara } \\
\text { Czarniawska \& Guje Sevón (1996) }\end{array}$ & $\begin{array}{l}\text { Ubertragung des Konzepts in die (neo-)institu- } \\
\text { tionalistische Organisationsforschung }\end{array}$ \\
Kjell Arne Røvik (2011) & Anwendung des Konzepts auf intraorganisationale Prozesse
\end{tabular}

\section{Neo-Liberalisierung}

Was kann unter den Prinzipien einer neo-liberalen Wirtschaft im Feld bildungspolitischen Policy Makings verstanden werden? Für die Beurteilung der Frage, ob es sich bei den von der OECD ausgehenden Prinzipien um solche handelt, die sich aus einer neoliberalen Logik generieren, wurde einschlägige Literatur aus der sozialwissenschaftlichen Globalisierungsforschung herangezogen, die sich vor allem mit der Weltwirtschaft und dem Kapitalismus seit den 1980er-Jahren befasst (vgl. u.a. Fourcade-Gourinchas \& Babb, 2002). 
Als besonders brauchbar für das hier behandelte Thema haben sich Texte von Nik Theodore und Jamie Peck und Kollegen herausgestellt (z.B. Peck et al., 2010), die strukturalistischen Ansätzen zur Erklärung von Neo-Liberalisierung und Neo-Liberalismus tendenziell kritisch gegenüberstehen. Sie gehen stattdessen davon aus, dass es sich hier um ein vielfältiges bzw. je nach Kontext variierendes Phänomen handelt. So wird Neo-Liberalisierung z.B. bei Brenner et al. (2010) als a variegated, geographically uneven and path-dependent process (S. 327) bezeichnet, mit entsprechenden kontextübergreifenden Effekten wie z.B. "geoinstitutionellen Differenzierungen". Aus diesem Texten lassen sich Kriterien entnehmen, wonach Neo-Liberalismus (neoliberalism) - verstanden als the ideologies and practices of freemarket capitalism (Brenner et al., 2010, S. 328) - und Neo-Liberalisierung (neoliberalization) - verstanden als [tendency] of regulatory change that have been unleashed across the global capitalist system since the 1970s (ebd., S. 329) - etwa mit Prinzipien wie Marktbasierung und Marktorientierung in Verbindung gebracht werden. An folgendem Fall zeigt sich, dass in den aus der OECD hervorgehenden Schriftstücken sich signifikant häufig jene Begriffe und Konzepte widerspiegeln, die in der herangezogenen Literatur zu Neo-Liberalisierung als typisch für ein marktorientiertes Denken eingestuft werden.

\section{Fallbeispiel: Entwicklung des Projekts Children and Youth at Risk}

Im Fokus der folgenden Betrachtung steht der schriftlich fixierte (und archivierte) Teil eines Interaktions- und Kommunikationsgeflechts zwischen der OECD und jenen Akteuren, die in den 1990er-Jahren bei der Entwicklung des Projekts Children and Youth at Risk beteiligt waren, bei dem das CERI der OECD die Federführung hatte. Das Projekt wurde im Verlauf der 1990er-Jahre mit dem Ziel durchgeführt, jenen Gruppen Zugang zu sowie gleichberechtigte Teilhabe an Bildungsangeboten zu gewähren, die als "most disadvantaged" galten. Hiermit waren insbesondere Menschen gemeint, die in Armut leben und ethnischen Minderheiten angehören, die also typischerweise aufgrund mehrerer sich überschneidender Merkmale (im hier diskutierten Fall ihrer klassenspezifischen, ethnischen und mitunter auch geschlechtlichen Gruppenzugehörigkeit) dem Risiko ausgesetzt waren, Opfer von Diskriminierung zu werden, educationally, socially, and economically. ${ }^{2}$ Mitglieder dieser Gruppe wurden ebenda identifiziert

$2 \quad$ Brief von Peter Evans (OECD) and Barbara B. Blum (Foundation for Child Development, New York), 
durch factors which are known to be highly predictive of educational failure. Der Autor des zitierten Briefes führte dazu aus, children who are especially affected would be those who are part of what may be called the long term poor. Dieser Adressatenkreis wurde in diesem Kontext kurz als at risk pupils bezeichnet ${ }^{3}$, wobei - wie im Folgenden zu sehen sein wird - die hier zusammenarbeitenden Akteure unterschiedliche Auffassungen zu den Fragen vertraten, worin das Risiko bestand, dem diese imaginierte Grossgruppe ausgesetzt war bzw. das mit dieser Gruppe assoziiert wurde und wie in diesem Zusammenhang Erfolg zu verstehen sei. Jedenfalls waren sich die Beteiligten einig, dass sich der Begriff "at risk" im Kontext des hier behandelten Projekts der OECD auf die gesamte Altersspanne von Heranwachsenden beziehe, den Übergang von Schule zum Arbeitsleben inbegriffen. Uneinigkeit bei der Rahmung von "Risiko" bestand allerdings schon insofern, als auf der einen Seite davon ausgegangen wurde, dass für diese Gruppe Risiko bestünde, und auf der anderen Seite die Meinung vertreten wurde, es würde ein Risiko von dieser Gruppe ausgehen, vielmehr gemeint im Sinne von risky und insofern als "Gefahr" für die Versorgungssysteme. So heisst es in einem mit Executive Summary überschriebenen Teil eines Dokuments aus dem Jahr 1993:

[S]ome children and youth come to be at risk of school failure and poor social insertion because of a cumulation of adverse events which place them at a significant learning disadvantage in contrast to other pupils. Poor nutrition, substanceabuse, non-supportive and even hostilefamilies, ineffectivemedical services, inflexible teachers and school curricula, poverty and prejudice are among the causal factors which have been identified. Furthermore, the threat to optimal development which such factors present will increase with time and if left untreated can lead to intractable and permanent disabilities. ${ }^{4}$ (CERI/ CD(93) 5, S.2)

Disabilities ist hier nicht im Sinne von "Behinderungen" zu verstehen, wie sie zu jener Zeit im Kontext von krankheitsbedingten Schädigungen und Beeinträchtigungen verstanden wurden, sondern als Konsequenzen von Benachteiligungen anderer Art. Vielmehr vollzog sich das Projekt "Children and Youth and Risk" in Äquivalenz zur sich im Kontext der UNESCO anbahnenden Education for All-Agenda, die paradoxerweise auf sämtliche Gruppen marginalisierter und vulnerabler Minderheiten abzielte, mit Ausnahme der Menschen mit Behinderungen (vgl. hierzu Kiuppis, 2014b). Der auf Children with Learning Difficulties August 1990.

3 Note by the Secretariat, OECD/CERI, Summary Report and Conclusions: Meeting of Country Representatives and Experts, April 1990, CERI/CY/90.14.

4 Note by the Secretariat, OECD Governing Board. März 1993, CERI/CD(93)5. 
bezogene Programmstrang findet auf Seiten der UNESCO seine Entsprechung wiederum im Programmstrang für Special Needs Education. Insofern waren beim «at risk»-Projekt Menschen mit gesundheitlichen Schädigungen, Beeinträchtigungen und Behinderungen eigentlich nicht mitgemeint, wobei allerdings die Trennlinie zwischen den imaginierten Populationen nicht immer scharf blieb - dies allein schon nicht aufgrund der Tatsache, dass manche Personen (z.B. Peter Evans, 1994) sowohl an dem "at risk"-Projekt als auch an anderen bildungspolitischen OECD-Initiativen zu Gunsten solcher Gruppen mitarbeiteten, die als marginalisiert beziehungsweise vulnerabel galten. Ein weiterer Grund für die zeitweise ungenaue Bestimmung der jeweiligen Adressatenkreise der Programme bzw. die Vermischung von an und für sich distinkten Fokusgruppen ist in der Individualisierung und Pathologisierung von at risk-Jugendlichen auf Seiten einzelner Mitgliedstaaten zu sehen (vgl. u.a. Wishart et al., 2006, S. 302).

Mit school failure war von der OECD eindeutig das Versagen von Kindern und Jugendlichen gemeint, wohingegen Akteure aus bestimmten Mitgliedsländern der OECD Schulversagen vielmehr als Mangel auf Seiten der Bildungsinstitutionen rahmten, so etwa in Kanada (vgl. Wotherspoon \& Schissel, 2001). Schulabbruch wurde hier darauf zurückgeführt, dass [s]chools can be risk-inducing phenomena in the cultural assumptions, classroom practices, and organizational and fiscal arrangements they adopt (ebd., S. 331).

Im oben zitierten Executive Summary heißt es weiter: In many OECD countries very substantial numbers of children are considered to be at risk, with the potential of being unable to contribute to the ever increasing exigencies of the labour market and thus contributing to growing social unease. ${ }^{5}$ Die Größe der "at risk"-Population beziffernd - wobei allerdings unklar ist, ob folgender vagen Formulierung dasselbe Verständnis von "at risk" zu Grunde lag -, heißt es an anderer Stelle:

Some $15-30 \%$ of children in many OECD countries may be identified as being at risk of failing at school and thus of jeopardising their successful transition to work and adult life. (Evans, 1994, S. 20)

5 Ebd. 
Für die Auswertung der Dokumente aus den Archiven der OECD stellte sich die Frage, mit welcher Motivation jeweilige Akteure bestimmte Projekte (wie z.B. das zur Analyse stehende) vorantrieben, die darauf abzielten, die Eingliederung von Children and Youth at Risk in Bildungssysteme und Arbeitsleben sicherzustellen. Ging es einem jeweiligen in den Archivquellen auftauchenden Akteur primär darum, dass dieser Gruppe die Chance zuteil werden mochte, Zugang zu Bildungsangeboten zu bekommen und daran gleichberechtigt teilzuhaben, oder standen vielmehr - wie es im letzten Zitat anklang - Befürchtungen im Vordergrund entsprechender Überlegungen, etwa dergestalt, dass der Übergang von Schule zu Arbeitsleben gefährdet sei oder, gar als Konsequenz der "totalen Exklusion" dieser Gruppe aus dem Bildungswesen (Ebersold, 1998, S. 5), Nachteile für die Versorgungssysteme gesehen wurden und somit - strukturell beurteilt - die Notwendigkeit gegeben war, diese Gruppe aufzufangen, da sie durch die Maschen zu fallen drohte? Hierauf deuteten z.B. Ausführungen der OECD hin, wonach there is a considerable need to find ways in which all children can be brought up and educated so that they may make their full contribution to the active society. (CERI/CD(89))

Darüber hinaus stellten sich bei der Analyse der Daten die Fragen, auf welcher Ebene es hier um das Anstreben von Erfolg ging und wie "gute Schulen" auszusehen hatten: im Besonderen auf der Ebene einzelner Individuen und ihrer Bedürfnisse oder im Allgemeinen im Hinblick auf Bedarfe auf der Systemebene? Je nach Rahmung der mit der Risikogruppe assoziierten Sozialen Probleme (etwa als "Vergeudung von Humankapital» oder als unrechtmässige "Ausgrenzung von Minderheiten") rekurrieren die verschiedenen in den Quellen sich widerspiegelnden Aussagen zum selben Thema auf unterschiedliche Sachverhalte, antizipieren folglich diverse imaginierte Lösungen bzw. Ziele und haben letztendlich konkurrierende Ansätze im Sinn.

Im Folgenden wird anhand der Archivmaterialien illustriert, dass die OECD in der Diskussion um Möglichkeiten des Gelingens von Integration von Children and Youth at Risk in Bildungssysteme rhetorisch in erster Linie darauf abzielte, unter Betonung von Prinzipien einer neo-liberalen Wirtschaft im Feld bildungspolitischen Policy Makings dafür zu werben, dass diese Gruppe nicht "erfolglos" bleibe. Aus den Daten geht hervor, dass 
diese auf marktorientierten Erwägungen basierende Idee auf der Ebene einzelner OECDMitgliedsländer in Verständnisse und Bedeutungen von at risk übersetzt wurde, die sich vielmehr die Rechte und Bedürfnisse einzelner Menschen zum Anlass nahm, um Wege zu finden und Möglichkeiten zu erarbeiten, diese in Bildungssysteme einzugliedern. Hierfür sollten auf Anregung der OECD zunächst solche innovativen Ansätze in Praxiskontexten ausfindig gemacht werden, [...] that are being tried out in order to help these children and young people make a success of their early lives and an effective transition to worthwhile employment. Brief von Peter Evans (OECD) an Barbara B. Blum (Foundation for Child Development, New York), August $1990 .^{6}$

\section{Diskursive Gleichzeitigkeit sich widersprechender Ideen}

Es sind in erster Linie systemische Erfordernisse, die von Seiten der OECD als Gründe angeführt werden, Children and Youth at Risk davor zu bewahren, in ihrer Bildungslaufbahn zu scheitern. Das Hauptanliegen des Projekts wurde darin gesehen, "to analyse the problems confronting the most disadvantaged and the re-shaping of the issues of access and equity in education and training in the mid-1990's in ways that affect broad cross-sections of our societies." ${ }^{7}$ Working Document, OECD Education Committee, Policy and Practice for Access, Participation, and Equity in Education: Developments, Approaches, and New Directions, Mai 1993, S. 2. Dieser Fokusgruppe als Kollektiv zu verhelfen, im Leben Erfolg zu haben, richtete sich auf Seiten der OECD nicht besonders deutlich an den Bedürfnissen der Menschen dieser Gruppe aus, sondern vielmehr, abstrahiert von Einzelfällen, an auf die Optimierung von Prozessen auf dem Arbeitsmarkt bezogenen Argumenten. Aus den Archivdaten wird deutlich, dass im Zusammenhang mit Themen wie z.B. curriculum reform, integrated services und "school management ${ }^{8}$ in OECD-Dokumenten klar marktorientierte Assoziationen deutlich werden, die allerdings in Kommunikationen mit Mitgliedsstaaten wie in einem Prisma gebrochen werden. Insofern rahmen widergespiegelte Positionen von Akteuren auf nationaler Ebene

6 Brief von Peter Evans (OECD) an Barbara B. Blum (Foundation for Child Development, New York), August 1990.

7 "Participation, Access and Equity", Working Document "Policy and Practice for Access, Participation, and Equity in Education: Developments, Approaches, and New Directions" vom Mai 1993.

8 Vgl. Dokument (September 1992) "OECD Study on Services integration for children and youth at risk and their families» mit Guidelines for case studies. 
dasselbe soziale Problem anders, wodurch es zu unterschiedlichen Bedeutungen von at risk und somit auch von hieran anschließenden Wörtern wie z.B. success kommt. Hier wird also deutlich, dass, wenn beispielsweise die Rede ist von "preventing vulnerable students from failing”, auf der Seite der OECD andere Rahmungen vorgenommen wurden als von Seiten der Mitgliedsländer. Diese Tendenz geht auch aus Forschungsergebnissen hervor, die im Rahmen von Analysen zur Konstruktion und Produktion einer at risk-Population im Jugendalter (Youth at Risk, YAR) erarbeitet wurden (Wishart et al., 2006).

An Archivmaterialien, die Einblick in die Vorbereitung, Durchführung und Nachbereitung jenes Treffens nationaler Vertreter/-innen und Expert/-innen geben, das am 17. und 18. Oktober 1989 im Hauptquartier der OECD in Paris stattfand, wird deutlich, dass auf Seiten der OECD stark vom Aspekt des Erfolgs ("pupil success") als antizipiertes Ziel für das Überleben des Systems ausgegangen wurde, wohingegen die am Projekt beteiligten Akteure auf Seiten der Mitglied-staaten, tendenziell eher auf den imaginierten Einzelfall bezogen, vom Erreichen individueller, jeweils selbst gesetzter Ziele ausgingen. In diesem Zusammenhang traf die OECD eine Wortwahl, die vor allem auf antizipierte "Effekte" auf Systemebene verwiesen, etwa the importance of the transition from pre-school to school [which/ that] can be crucial to a child's successful adaptation"; effective socialisation; effective management; effective provision; effective practice ${ }^{9}$; optimal progress; pupil progress; successful provision; school management; management for change; and "performance based accountability. ${ }^{10}$ Im Kontext von "parental and community involvement" wird hier z.B. die Rolle von "business services" angesprochen und im Vordergrund solcher Überlegungen stehen Fragen wie "Are some systems more effective than others?" Es geht in erster Linie darum, ...to prevent drop out and help students at risk to remain longer in school and to begin to achieve much higher standards of performance (ebd.). ${ }^{11}$ In diesem Zusammenhang gab das CERI der OECD eine grosse Anzahl von Fallstudien in Mitgliedsländern in Auftrag, ...that cover a wide range of issues such as curriculum development and the teaching of basic skills, methods of reducing truancy rates and

9 Brief von Peter Evans (OECD) an Aletta Grisay (Service de Pedagogie Experimentale, Liège), Oktober 1990. 10 vgl. z.B. das zitierte Dokument von September 1992, S. 3.

11 Proposal for an international study on services integration for primary aged children and youth at risk and their families, September 1992. 
drop out, special strategies for minority groups, school organization and management, parent and community relations, transition to work and services integration. ${ }^{12}$

Aus einem mit Summary of the Main Conclusions betitelten Dokument, das im Nachgang zum Treffen im Oktober 1989 verfasst wurde und mit dem Dokument "Notes for the Paris meeting on Oct 17-18, 1989, OECD Governing Board, November 1989, CERI/CD(89)17» kursierte, wird als Kontrast zu den oben angedeuteten Positionen der OECD das kollektive Ansinnen der Teilnehmer/-innen zusammenfasst, dass dieses Projekt weniger korrektiv ansetzen möge, denn vielmehr langfristig und prozessorientiert auszurichten sei:

It is now widely held that educational disadvantage that may stem from social disadvantage cannot be overcome simply through interventions, either individual or systemic, at only one stage of the child's life but that these need to be extended throughout the whole of the pre-school and school years. For this reason the meeting endorsed the view that the project concerns should cover the whole of the period from birth to the transition to work as originally proposed S. $2 .^{13}$

Im März des Jahres 1993 fand im Hauptsitz der OECD in Paris ein Treffen statt, an dem Repräsentantinnen und Repräsentanten von 12 Mitgliedstaaten, acht Experten und zwei Stiftungen (SVO = Foundation for Educational Research aus Holland und die Charles Stewart Mott Foundation aus den USA) teilnahmen. Hier wurde das Projekt vorge-stellt sowie sein thematischer Hintergrund diskutiert. Ausserdem wurde beschlossen, 1993 Europa zu besuchen, Nord-Amerika 1994 und Australien und Pazifik 1995. In diesem Kontext wird deutlich, dass die OECD (wie es etwa in Executive Summaries oder Notes by the Secretariat zu ersehen ist) ihre Ideen an der Systemebene festmacht, wobei von Seiten der Mitgliedsstaaten eher die Rede ist von needs of individuals. Während auf der einen Seite 'Selektion' und 'Wettbewerb' als neue, vor allem von der OECD ausgehende Prinzipien stehen, werden diese Ideen auf der anderen Seite (in den Mitgliedsländern) in die Rede von «besonderen pädagogischen Bedarfen» übersetzt.

Aus dem Dokument mit dem Titel Proposal for an international study on services integration for primary aged children and youth at risk and their families, das mit dem Projekt Children and

\begin{tabular}{ll}
\hline 12 & Ebd \\
13 & (CERI/CD (89)17)..
\end{tabular}


Youth at Risk im Zusammenhang stand, geht unter dem Punkt "Overall Goal» (S. 1) folgende Formulierung hervor: The overall goal of this study is to gather information and data to inform governments and others on the critical issue of integrated services for solving crises in OECD countries for children and youth at risk and their families" und beim Punkt "Rationale (ebd.) heisst es: "improving life outcomes for children, youth and families". ${ }^{14}$ Hier ist klar zu erkennen, dass es der OECD in erster Linie darum ging, Interventionen "outcome»-orientiert anzugehen und situativ Schaden abzuwenden, wohingegen sich Projektpartner aus den Mitgliedstaaten tendenziell eher für prozessorientierte, die längerfristige Partizipation von Menschen sicherstellende Unterstützungsmaßnahmen aussprachen. Die OECD ging dabei vom Sozialen Problem hoher Arbeitslosigkeit in OECD-Mitgliedsländern aus und definierte children who are at risk anderswo als They are at risk of failing to develop their full potential, thereby making substantially less of a contribution to society than might otherwise be the case [Hervorhebung: der Autor]. ${ }^{15}$

Zwei Monate später gingen aus einem Memorandum und anhängenden Mission Report eines damals führenden OECD-Vertreters weitere Details zu diesem Meeting hervor. ${ }^{16}$ Hier ist interessant, dass die Frage aufgeworfen wurde, «how much equity do we want?». In diesem Zusammenhang wurde zwischen der OECD und den Mitgliedsländern diskutiert, wie weit "the alignment of society interests with those of individuals/individual parents" gehen sollte. Hier kam es also zu einer Debatte, die sich um die Diskrepanz drehte, that the collective implicit and direct costs of failure within the school-age population (noted by the Secretary General) are not fully perceived by individual (middle class?) parents who seek rather to maintain an advantage for their children (ebd.).

\section{Schluss}

Dieser Beitrag baut konzeptionell auf einem Begriff von «Übersetzung» auf, mit dem nicht linguistisch Zwei- bzw. Mehrsprachigkeit assoziiert wird, sondern die Weitergabe und

\footnotetext{
14 (doc vom September 1992, S. 3).

15 Vgl. z.B. S. 2 des Working Document "Policy and Practice for Access, Participation, and Equity in Education: Developments, Approaches, and New Directions" vom Mai 1993.

16 Memorandum / Mission Report von Alan Wagner (OECD), versandt an Peter Evans im Juli 1993.
} 
Transformation von Wissensbeständen zwischen Organisationen bzw. mit dem, in Anlehnung an das Begriffsverständnis des Norwegischen Sozialwissenschaftlers Kjell Arne Røvik, ein entsprechender Prozess innerhalb von Organisationen gemeint ist. Der Text basiert auf einem zugleich organisations- und wissenssoziologischen Zugang zum Thema, bei dem es in erster Linie um - je nach Akteursgruppe unterschiedliche - "sprachliche Verpackung" desselben bildungspolitischen Projektes geht (vgl. Røvik, 2011, S. 643). Hier wird angenommen, dass Ideen bei ihrer Weitergabe von Akteur zu Akteur sowie bei an diesen Transfer anschliessenden Implementierungsprozessen in Transformation begriffen sind und ein jeweiliges Verständnis einer Idee aus den Handlungen einer Reihe von Akteuren resultiert, in der jeder Akteur bestimmte Wörter entsprechend seiner eigenen Projekte "übersetzt» und gebraucht, es also zeitgleich zu einer Vielzahl unterschiedlicher Versionen und sprachlicher Rahmungen desselben Sachverhalts kommt. Unter Verweis auf dasselbe beziehen sich verschiedene Akteure im Verlauf dieser Prozesse also auf Verschiedenes, und die Gleichzeitigkeit unterschiedlichen Gebrauchs ein und desselben Wortes (z.B. at risk; success) wird angenommen als [resulting] from the actions of a chain of agents each of whom translates it in accordance with his/her own projects (Latour, 1986, S. 264). Bezogen auf das hier behandelte Fallbeispiel stellt sich das so dar, dass zwar sämtliche an den Abstimmungs- und Entscheidungsprozessen beteiligten Akteure einig sind, welche Populationen als "at risk" zu klassifizieren sind, dass aber kein Konsens in der Frage besteht, worin genau das Risiko bestehe. Insofern trifft in diesem Fall jener Umstand zu, den der deutsche Bildungswissenschaftler Frank-Olaf Radtke am Beispiel von best practices so formuliert, es würden - im hier vorliegenden Fall von Seiten der OECD - Lösungen erarbeitet, bebei denen nicht klar sei, zu welchen lokalen Problemen sie passten (Radtke, 2009, S. 164). Hier wird deutlich, dass es der OECD nicht in erster Linie darum ging, Konsens im jeweiligen Verständnis von at risk zu erlangen (etwa indem sie sich des Instruments der Überzeugung zu bedienen versucht), sondern darum, Übereinstimmung in der Frage zu erzielen, dass das Projekt wichtig sei.

Die in diesem Kontext analysierten Übersetzungen und damit einhergehenden Missinterpretationen finden sich nicht nur hier, sondern auch bei anderen Themen ähnlich wieder. So deutet vor allem U.S.-amerikanische Literatur auf äquivalente Phänomene hin, etwa Ayers und Carlone (2007) auf die Manifestierung neoliberaler Diskurse innerhalb 
eines sogenannten Job- Trainingsprogramms; Finn et al. (2010) auf entsprechende Prozesse im Zusammenhang mit dem Personal Responsibility and Work Opportunity Reconciliation Act; und kürzlich, im Nachgang zur Finanzkrise, Wilkins (2013) auf Übersetzungen neoliberaler Policy-Ideen im Rahmen von Abwägungen bei der Schulwahl. In all diesen Untersuchungen stellt sich die Tendenz heraus, dass sich trotz des im Rahmen von Abstimmungs- und Entscheidungsprozessenvermeintlich dominierenden Konsensesin BezugaufbestimmteSachverhalte, der Gebrauch zentraler Wörter - und somit, Wittgenstein zufolge, ihre Bedeutung - durch jene Akteure, die auf nationaler Ebene zu "Weltprogrammatiken" Stellung beziehen, deutlich von solchen Vorgaben unterscheidet, die in diesem Zusammenhang von den jeweils federführenden Internationalen Organisationen gemacht wurden. Während die OECD im hier präsentierten Fall für die Rahmung der konzeptionellen Grundzüge des Projekts Children and Youth at Risk eine Sprache wählt, die sich an neo-liberalen Prinzipien (z.B. Wettbewerb und Markt) orientiert, werden anhand von Positionierungen von Akteuren aus den OECD-Mitgliedsländern mitunter ganz anders gelagerte Motivationen für die Durchführung dieses Projekts deutlich, etwa die Sicher-stellung von Bildungsgerechtigkeit und damit einhergehend die Betonung besonderer pädagogischer Bedürfnisse bestimmter Gruppen von Kindern und Jugendlichen.

Gestützt durch theoretische Annahmen aus der neo-institutionalistischen Organisationsforschung zur "Übersetzung» von Ideen sowie zur Neo-Liberalisierung, skizziert der vorliegende Beitrag einen Fall, bei dem sich die Aushandlung der Grundzüge des Projekts Children and Youth at Risk für benachteiligte Kinder und Jugendliche zwischen dem CERI der OECD und ihrem Umfeld abspielt und Positionen dritter Akteure zum selben Thema inhaltlich/auf der Bedeutungsebene einen Kontrast zu den Dokumenten der OECD darstellen. Bei der Kontrastierung der Positionen wird deutlich, dass jene Prinzipien einer neoliberalen Wirtschaft, die z.B. Wettbewerb und Markt in den Fokus der Aufmerksamkeit rücken, zwar in den von der OECD autorisierten Projektskizzen enthalten sind, sich aber nicht (mehr) in jenen Dokumenten widerspiegeln, die von anderen Akteuren zurückgespielt werden. In diesem Kontext fällt also beim Quellenstudium auf, dass von Seiten der OECD neo-liberale Prinzipien in den Diskurs um Bildungsangebote für marginalisierte Minderheiten eingebracht wurden, die aber in jenen Konstruktionen der anderen am Projekt beteiligten 
Akteure, die sowohl von konkret praktizierten Vorbildern abstrahieren als auch von wünschenswerten Idealvorstellungen gespeist sind, keine Rolle mehr spielen. Den diesem Artikel zu Grunde liegenden theoretischen Annahmen zufolge wurden die neo-liberalen Ideen der OECD (etwa zu Wettbewerb und Markt) durch verschiedene Akteure auf lokaler Ebene, etwa in Entsprechung zu jeweils eigenen Projekten- übersetzt und dabei transformiert. Diese Prozesse vollziehen sich vor allem auf der Handlungsebene der anderen Akteure, die das Wissen gemäss den ihren jeweils eigenen Projekten zu Grunde liegenden «institutionellen Logiken» verändern (vgl. hierzu Kiuppis 2014a). Am hier skizzierten Fall zeigt sich, dass jene Prinzipien einer neo-liberalen Wirtschaft, die von der OECD in Projekte eingebracht werden, von anderen an diesem Projekt beteiligten Akteuren "übersetzt» und insofern jeweiligen Bedarfslagen angepasst werden. In Bezug auf das im vorliegenden Artikel behandelte Thema und den hier gewählten theoretischen Bezugsrahmen lässt sich in diesem Zusammenhang Literatur aus dem Überschneidungsbereich zwischen Organisationsforschung und der Analyse Sozialer Bewegungen anführen. Bei Campbell heisst es hierzu:

[As] neoliberal ideas diffused among countries during the 1980's and
$1990^{\prime}$ 's, individual governments tended to adopt different elements of the
neoliberal model and blend them into their already existing institutional
traditions in ways that resulted in less convergence and isomorphism than
most diffusion theorists might expect (Campbell, 2005, S. 55; vgl. hierzu
auch Campbell \& Pedersen, 2001). Aber auch neuere Literatur verweist
auf solche Prozesse, in deren Rahmen ein Markt- oder neoliberales
Modell im Zusammenhang mit Lernen und Ergebnismessung bis
heute von Internationalen Regierungsorganisationen ausgehe und
entsprechende Diskurse in Mitgliedstaaten dominiere (so etwa
Desjardins, 2013; Sellar \& Lingard, 2013; Morley et al., 2014). Innerhalb
der EU spiegelt sich der neoliberale Diskurs und die Semantik der OECD
nach wie vor allem in Bezug auf die arbeitsmarktpolitische Ausrichtung
der Bildungsprogramme wider (Fuchs, 2011, S. 272). Während dem
Sachverhalt Children and Youth at Risk seit den 1990er-Jahren neue
Bedeutungen eengehaucht wurden, besteht das Soziale Problem als
solches unverändert fort - auch noch immer in OECD-Mitgliedstaaten.

\section{Literatur}

Ayers, D.F. \& Carlone, D. (2007). Manifestations of neoliberal discourses within a local jobtraining program. International Journal of Lifelong Education, 26(4), 461-479.

Ball, S. J. (1998). Big policies/small world: An introduction to international perspectives in education policy. Comparative Education, 34 (2), 119-130. 
Brenner, N., Peck, J. \& Theodore, N. (2010). After neoliberalization? Globalizations, 7(3), 327-345

Broadfoot, P. (2000). Comparative education for the 21 st century: retrospect and prospect. Comparative Education, 36 (3), 357-371.

Callon, M. (1975). Les Opérations de Traductions. In C. Gruson, P. Roqueplo, P. Thuillier (Éds.). Incidence des Rapports Sociaux sur le Développement Scientifique. Paris: Comité d'organisation des recherches appliquées sur le Développement économique et social.

Callon, M. (1986). Éléments pour une sociologie de la traduction: la domestication des coquilles Saint-Jacques et des marins-pêcheurs dans la baie de Saint-Brieuc. L'Année sociologique (1940/1948-), 169-208.

Campbell, J. L. (2005). Where do we stand? Common mechanisms in organizations and social movements research. In G. F. Davis, D. McAdam, W. R. Scott \& M. N. Zald (Eds.), Social Movements and Organization Theory (pp. 41-68). Cambridge: Cambridge University Press.

Campbell, J. L. \& Pedersen, O. K. (2001). The second movement in institutional analysis. In J. L. Campbell \& O. K. Pedersen, O. K. (Eds.), The rise of neoliberalism and institutional analysis (pp. 249-282). Princeton, N.J.: Princeton University Press.

Carnoy, M. \& Rhoten, D. (2002). What does globalization mean for educational change? A comparative approach. Comparative Education Review, 46(1), 1-9.

Caruso, M. (2008). World systems, world society, world polity: theoretical insights for a global history of education. History of Education, 37(6), 825-840.

Centeno, V. (2011). Lifelong learning: a policy concept with a long past but a short history. International Journal of Lifelong Education, 30(2), 133-150.

Cowen, R. (2009). The transfer, translation and transformation of educational processes: and their shape shifting? Comparative Education, 45 (3), 315-327.

Czarniawska, B. \& Joerges, B. (1996). Travels of ideas. In B. Czarniawska \& G. Sevón (Eds.), Translating Organizational Change (pp. 13-48). Berlin: de Gruyter.

Czarniawska, B. \& Sevón, G. (1996). Translating Organizational Change. Berlin: de Gruyter.

Dale, R. (2012). Global Education Policy: Creating Different Constituencies of interest and different modes of valorisation. In A. Verger, H. K. Altinyelken \& M. Novelli (Eds.), Global Education Policy and International Development: New Agendas, Issues and Policies (pp. 279- 288). New York: Bloomsbury.

Desjardins, R. (2013). Considerations of the impact of neoliberalism and alternative regimes on learning and its outcomes: an empirical example based on the level and distribution of adult learning. International Studies in Sociology of Education, 23(3), 182-203. 
Ebersold, S. (1998). Exclusion and Disability. Paris: OECD, http://www1.oecd.org/edu/ innovation-education/1856907.pdf

Evans, P. (1994). Tackling Educational Disadvantage. OECD Observer, 186, 20-22.

Finn, J. L., Nybell, L. M. \& Shook, J. J. (2010). The meaning and making of childhood in the era of globalization: Challenges for social work. Children and Youth Services Review, $32(2), 246-254$.

Fourcade-Gourinchas, M. \& Babb, S. L. (2002). The Rebirth of the Liberal Creed: Paths to Neoliberalism in Four Countries. American Journal of Sociology, 108 (3), 533-579.

Fuchs, E. (2011). Bildung im Zeichen der Globalisierung. In G. Mertens, U. Frost, W. Böhm, L. Koch \& V. Ladenthin (Hrsg.), Allgemeine Erziehungswissenschaft II (S. 263-274), Paderborn: Ferdinand Schöningh.

Gherardi, S. \& Nicolini, D. (2000). The organizational learning of safety in communities of practice. Journal of Management Inquiry, 9 (1), 7-18.

Hasse, R. \& Krücken, G. (2009). Neo-institutionalistische Theorie. In G. Kneer \& M. Schroer (Hrsg.), Handbuch Spezielle Soziologien (S. 237-251). Wiesbaden: VS Verlag für Sozialwissenschaften.

Jakobi, A. P. (2007). Die Bildungspolitik der OECD: Vom Erfolg eines scheinbar machtlosen Akteurs. Zeitschrift für Pädagogik, 53(2), 166-181.

Jonsson, S. (2009). Refraining from imitation: Professional resistance and limited diffusion in a financial market. Organization Science, 20(1), 172-186.

Kiuppis, F. (2007). Zur Erweiterung der Klassifizierung gesundheitsbezogener Daten auf Behinderung: Fallstudie über die Konstruktion und "Institutionalisierung" von Paradigmenwechseln durch internationale Organisationen. Aachen: Shaker.

Kiuppis, F. (2013). Why (not) associate the principle of inclusion with disability? Tracing connections from the start of the 'Salamanca Process'. International Journal of Inclusive Education, (ahead-of-print), 1-16.

Kiuppis, F. (2014a). Heterogene Inklusivität, inklusive Heterogenität: Bedeutungswandel imaginierter pädagogischer Konzepte im Kontext Internationaler Organisationen. Münster/ New York: Waxmann.

Kiuppis, F. (2014b). Why (not) associate the principle of inclusion with disability? Tracingconnections from the start of the 'Salamanca Process'. International Journal of Inclusive Education, 18(7), 746-761.

Krippendorff, K. (2012). Content analysis: An introduction to its methodology. Beverly Hills, CA: Sage.

Latour, B. (2005). Reassembling the Social - An Introduction to Actor-Network-Theory. Oxford: Oxford University Press. 
Latour, B. (1986). The powers of association. In J. Law (Ed.), Power, action and belief: A new sociology of knowledge (pp. 264-280). London: Routledge.

Law, J. (1992). Notes on the theory of the actor-network: ordering, strategy, and heterogenity. Systems Practice, 5(4), 379-393.

Mausethagen, S. (2013). Governance through concepts: The OECD and the construction of "Competence" in Norwegian education policy. Berkeley Review of Education, 4(1), 161-181.

Morley, L., Marginson, S. \& Blackmore, J. (2014). Education and neoliberal globalization. British Journal of Sociology of Education, 35(3), 457-468.

Nóvoa, A. N. \& Yariv-Mashal, T. (2003). Comparative research in education: A mode of governance or a historical journey? Comparative Education, 39(4), 423-438.

Ozga, J. (2005). Modernizing the education workforce: a perspective from Scotland. Educational Review, 57(2), 207-219.

Peck, J., Theodore, N. \& Brenner, N. (2010). Postneoliberalism and its malcontents. Antipode, 41(1), 94-116.

Phillips, D. \& Ochs, K. (Eds.). (2004). Educational policy borrowing: Historical Perspectives. Oxford: Symposium books.

Radtke, F. O. (2009). Evidenzbasierte Steuerung. Der Aufmarsch der Manager im Erziehungssystem. In R. Tippelt (Hrsg.), Steuerung durch Indikatoren (S. 157-180). Opladen: Budrich.

Rinne, R. \& Ozga, J. (2011). Europe and the global: The role of the OECD in Education politics. In J. Ozga, P. Dahler-Larsen \& H. Simola (Eds.), Fabricating quality in education: Data and governance in Europe (pp. 66-75). London: Routledge.

Robertson, S. L. (2012). Researching Global Education Policy: Angles In/On/Out. In Verger, H. K. Altinyelken \& M. Novelli (Eds.), Global Education Policy and International Development: New Agendas, Issues and Policies (pp. 33-52). New York: Bloomsbury.

Roldán-Vera, E. R. \& Schupp, T. (2006). Network analysis in comparative social sciences. Comparative Education, 42(3), 405-429.

Røvik, K. A. (2011). From fashion to virus: An alternative theory of organizations' handling of management ideas. Organization Studies, 32(5), 631-653.

Scheuer, J. D. (2006). Grounding New Institutional Theory on a micro-sociological and practicebased foundation - exploring models of translation. Vortrag im Rahmen des 22. EGOS Colloquiums in Bergen.

Schriewer, J. (2007). Weltkultur und kulturelle Bedeutungswelten. Zur Globalisierung von Bildungsdiskursen. Frankfurt am Main/ New York: Campus. 
Schulte, B. (2008). Zur Rettung des Landes. Bildung und Beruf im China der Republikzeit. Frankfurt am Main: Campus.

Scott, W. R. (1994). Institutions and organizations: toward a theoretical synthesis. In W. R. Scott \& J. W. Meyer (Eds.), Institutional environments and organizations: Structural complexity and individualism (pp. 55-80). Thousand Oaks, CA: Sage.

Sellar, S. \& Lingard, B. (2013). The OECD and global governance in education. Journal of Education Policy, 28(5), 710-725.

Serres, M. (1974). Hermès III: la traduction. Paris: Editions de Minuit.

Silova, I. (2004). Adopting the language of the new allies. In G. Steiner-Khamsi (Ed.), The global politics of educational borrowing and lending (pp. 75-87). New York: Teachers College Press.

Steiner-Khamsi, G. (2006). The economics of policy borrowing and lending: A study of late adopters. Oxford Review of Education, 32 (5), 665-678.

Steiner-Khamsi, G. \& Quist, H. O. (2000). The politics of educational borrowing: Reopening the case of Achimota in British Ghana. Comparative Education Review, 44(3), 272-299.

Steiner-Khamsi, G. \& Waldow, F. (Hrsg.) (2012). World Yearbook of Education 2012: Policy Borrowing and Lending in Education. New York: Routledge.

Strang, D. \& Soule, S. A. (1998). Diffusion in organizations and social movements: From hybrid corn to poison pills. Annual Review of Sociology, 24, 265-290.

Takayama, K. (2012). Exploring the interweaving of contrary currents: transnational policy enactment and path-dependent policy implementation in Australia and Japan. Comparative Education, 48(4), 505-523.

Theodore, N. \& Peck, J. (2012). Framing neoliberal urbanism: Translating 'commonsense' urban policy across the OECD zone. European Urban and Regional Studies, 19 (1), 20-41.

Verger, A., Altinyelken, H. K. \& de Koning, M. (2013). Global Education Reforms and the New Management of Teachers: A Critical Introduction. In A. Verger, H. K. Altinyelken \& M. de Koning (Eds.), Global Managerial Education Reforms and Teachers (pp. 1-18). Amsterdam: Education International Research Institute.

Waldow, F. (2009). Undeclared imports: silent borrowing in educational policy making and research in Sweden. Comparative Education, 45(4), 477-494.

Wilkins, A. (2013). Libertarian paternalism: policy and everyday translations of the rational and the emotional. Critical Policy Studies, 7(4), 395-406.

Winker, G. \& Degele, N. (2009). Intersektionalität. Zur Analyse sozialer Ungleichheiten. Bielefeld: transcript. 
Wishart, D., Taylor, A. \& Shultz, L. (2006). The construction and production of youth 'at risk'. Journal of Education Policy, 21 (03), 291-304.

Wittgenstein, L. (2001 [1936-1946]). Philosophische Untersuchungen. In J. Schulte (Hrsg.), Philosophische Untersuchungen. Frankfurt am Main: Suhrkamp.

Wotherspoon, T. \& Schissel, B. (2001). The business of placing Canadian children and youth 'at risk'. Canadian Journal of Education, 26 (3), 321-339.

Wright, S. (1998). The politicization of 'culture'. Anthropology today, 14(1), 7-15. 